\title{
Semantic Features Reveal Different Networks During Word Processing: An EEG Source Localization Study
}

\author{
Mansoureh Fahimi Hnazaee ${ }^{*}$ Elvira Khachatryan and Marc M. Van Hulle ${ }^{\dagger}$ \\ Laboratory for Neuro- and Psychophysiology, Department of Neurosciences, KU Leuven, Leuven, Belgium
}

OPEN ACCESS

Edited by:

Arthur M. Jacobs,

Freie Universität Berlin, Germany

Reviewed by:

Arash Aryani,

Freie Universität Berlin, Germany Lars Kuchinke

International Psychoanalytic University

Berlin, Germany

*Correspondence:

Mansoureh Fahimi Hnazaee mansoureh.fahimihnazaee@kuleuven.be

${ }^{+}$Marc M. Van Hulle orcid.org/0000-0003-1060-7044

Received: 03 October 2018 Accepted: 29 November 2018 Published: 13 December 2018

Citation: Fahimi Hnazaee M, Khachatryan E and Van Hulle MM (2018) Semantic Features Reveal Different Networks During Word Processing: An EEG

Source Localization Study.

Front. Hum. Neurosci. 12:503. doi: 10.3389/fnhum.2018.00503
The neural principles behind semantic category representation are still under debate. Dominant theories mostly focus on distinguishing concrete from abstract concepts but, in such theories, divisions into categories of concrete concepts are more developed than for their abstract counterparts. An encompassing theory on semantic category representation could be within reach when charting the semantic attributes that are capable of describing both concept types. A good candidate are the three semantic dimensions defined by Osgood (potency, valence, arousal). However, to show to what extent they affect semantic processing, specific neuroimaging tools are required. Electroencephalography (EEG) is on par with the temporal resolution of cognitive behavior and source reconstruction. Using high-density set-ups, it is able to yield a spatial resolution in the scale of millimeters, sufficient to identify anatomical brain parcellations that could differentially contribute to semantic category representation. Cognitive neuroscientists traditionally focus on scalp domain analysis and turn to source reconstruction when an effect in the scalp domain has been detected. Traditional methods will potentially miss out on the fine-grained effects of semantic features as they are possibly obscured by the mixing of source activity due to volume conduction. For this reason, we have developed a mass-univariate analysis in the source domain using a mixed linear effect model. Our analyses reveal distinct networks of sources for different semantic features that are active during different stages of lexico-semantic processing of single words. With our method we identified differences in the spatio-temporal activation patterns of abstract and concrete words, high and low potency words, high and low valence words, and high and low arousal words, and in this way shed light on how word categories are represented in the brain.

Keywords: high-density EEG, source localization, semantic category representation, single word processing, linear mixed effect model

\section{INTRODUCTION}

Different cortical areas are involved in representing semantic categories and concepts, collectively known as the "semantic system" (Binder et al., 2009), and word representation ("semantic map") has recently been mapped comprehensively with fMRI (Huth et al., 2016). Much of our understanding comes from the vast amount of neuroimaging and electrophysiological data recorded from both healthy subjects and neurogenerative disorder patients as well as the occasional invasive recording in patients during or in the work-up of surgery. However, despite this evidence, it is still not clear on what basis these representations differ. 
One of the most prominent theories of semantic word processing is the grounded cognition or embodied cognition model. This model suggests that semantic knowledge resides in high-level perception and motor representation systems (Barsalou, 2008, 2010). In other words, a word is comprehended based on the modality-specific neural systems (for example, visual features such as color, shape and motor actions) that are associated with the definition of the word. Indeed, a number of fMRI studies have demonstrated that, for example, animate objects tend to cluster in the lateral part of the fusiform gyrus while inanimate or manmade objects cluster in the medial part (Martin and Chao, 2015). Furthermore, it has been shown that certain category mappings are based on specific shapes, such as "face" and "body" patches (Tsao et al., 2008). However, the embodied cognition framework has received considerable criticism, too (Mahon and Caramazza, 2008). For example, a number of lesion studies have been reported where damage to the brain's modal system led to category-specific deficits and to disproportionately preserved categories such as animals, foods, or artifacts (Barsalou et al., 2003; Caramazza and Mahon, 2003). An attempt to explain this along the lines of embodied cognition would need to resort to the most prevalent modality behind these categories, e.g. artifacts separated from animals and food as they call upon systems involved in manipulation or the action in response to experiencing the word, as evidenced by a larger activation in the left premotor cortex and the pre- and post-central gyrus (Hwang et al., 2009). However, category-specific deficits are hard to explain when relying only on embodied cognition, since many modality-specific systems might overlap between categories, and a single category might contain several distinct modality-specific features. This raises the question of how these modality-specific features are linked within one category and, conversely, when they span several categories, how they can be distinguished between categories. Increasing evidence suggests the existence of a modality-invariant integrative mechanism in addition to the modality-specific neural systems described in the classical model. This integrative component is called the semantic "hub," and modality-specific neural systems are referred to as the "spokes" of the model. The hub and spoke model is regarded as the middle ground between the embodied and disembodied hypotheses (Mahon and Caramazza, 2008; Lambon Ralph, 2013).

However, the grounded cognition model, with its hub and spoke extension model (Lambon Ralph, 2013), can be criticized from several angles. First of all, modality-specific features of a concept are much less variant between different subjects when shown as a clear visual stimulus (image). However, when stimuli are conceptual in nature (such as words presented in written or spoken form), the perceptual and motor-sensory functions they refer to are much more difficult to control as the experience designated to the specific entities is to a large degree subject-dependent (Kemmerer, 2014). Secondly, which is particularly relevant to our study, the grounded cognition model only explains features that are rooted in the physical experience with the concept, limiting its applicability to concrete concepts. However, abstract concepts such as "democracy" or "emotions" are not explained by this theory, despite of their prominent presence in human language (Kemmerer, 2014).

The difference between processing abstract and concrete words has been addressed by a number of theories, the main ones being the dual coding and context availability theories (Kounios and Holcomb, 1994; Wang et al., 2010). The dual coding theory claims that two separate systems reside in the brain: a nonverbal "imagery" system that implements the modality-specific aspects of a concept (similar to the grounded cognition model), and a purely verbal "linguistic" system that is involved in the abstract form of language. According to the context availability theory, the processing of concrete and abstract words rarely occurs in isolation, but rather in the context in which they are understood. Since the context of concrete words is mainly constrained by the words' physical properties, understanding these words will again involve modality-specific brain systems as defined in the grounded cognition model. In the case of abstract concepts, however, context is more variable and experience-dependent.

Both theories put the grounded cognition model for different categories of concrete words into a larger framework that would also include abstract concepts. For example, some studies have attempted to define the "hub" of the previously mentioned hub and spoke model, and considered the anterior temporal lobes as a pan-modal hub for all concepts (so as to also include abstract concepts; Hoffman and Ralph, 2018). However, none of the aforementioned theories discuss the possibility of also having categories for abstract concepts and considers them homogeneous, despite the gamut of semantically significant subdivisions such as numbers, emotions, etc. The question remains whether an embodying ground can be defined for abstract concepts by means of well-defined domains. Despite some efforts in this regard (defining different abstract categories such as numbers and emotions; Kemmerer, 2014), we believe a good approach would be to search for a semantic space that can describe both abstract and concrete words. An important set of semantic attributes are the three dimensions according to the semantic differential scale proposed by Osgood and colleagues (Osgood et al., 1975). According to the study by Osgood, the affective meaning of linguistic terms can be quantified in three independent dimensions marked by the following polar adjectives: "evaluation" or "valence" (good- bad, pleasantunpleasant, positive-negative), "potency" (strong-weak, heavystrong, large-small), and "activity," also called "arousal" in most current models (active-passive, fast-slow, sharp-dull) (Fontaine et al., 2013) thereby avoiding possible confusion with motor activity. These semantic attributes proved to be robust across age groups, cultures, and languages. Specifically, the neural correlates of valence and arousal for word processing have been investigated extensively in the past decade in the framework of emotion word processing (Kuchinke et al., 2005; Kissler et al., 2006; Lewis et al., 2007; Citron et al., 2013). For example, valence has already been suggested to ground abstract concepts in the same neural systems underlying basic emotions (Kousta et al., 2011; Vigliocco et al., 2014) since there seems to be a high level of interaction between abstractness and valence. In this study we extend this research by including the two other Osgood dimensions: arousal and potency. To the best of our knowledge, we are 
the first to propose these dimensions to dissociate their neural representation from that of abstract and concrete concepts. In order to unveil the networks activated by the Osgood dimensions, but also by abstract vs. concrete categories, and to detect any differences in the evoked spatio-temporal activity patterns, we need tools that match the temporal resolution of the underlying neural mechanisms. The temporal course of word processing is crucial for the functional interpretation of brain activity, e.g., to determine during which process we can see the effect of lexical or semantic properties.

A crucial limitation of all the aforementioned theories is that they have predominantly relied on functional neuroimaging techniques such as $\mathrm{PMRI}$ and PET, which are limited in temporal resolution (Bookheimer, 2002). As word recognition involves stages that unfold on a scale of milliseconds, such as visual encoding, lexical activation, and semantic presentation (Laszlo and Federmeier, 2014), the temporal resolution should be on par with the dynamics of these processes. Whether these stages are sequential or partially parallel and interactive is still debated (Hauk et al., 2006; Grainger and Holcomb, 2009), however it is clear that hemodynamic imaging cannot track the activity progression or resolve differences in activity patterns between words (Helenius et al., 1998; Bentin et al., 1999). On the other hand, studies that involve lesions and electrical stimulations do not provide the proper scope for the large-scale networks that are possibly involved.

By virtue of its excellent temporal resolution, the EEG technique has been hailed for gauging the brain's semantic processes. Studies using EEG/ERP recordings have successfully distinguished differences in word categories in different stages of semantic processing, starting from detecting differences in word length and word frequency around 100 and $200 \mathrm{~ms}$ (Hauk et al., 2006) to the distinction in processing semantic categories, such as abstract vs. concrete concepts (Bentin et al., 1999), animals vs. tools (Simanova et al., 2010) and more generally natural objects vs. artifacts (Kiefer, 2001), around 400-500 ms (N400 potential).

The main drawback of traditional scalp EEG is its relatively low spatial resolution, which falls short in detecting differences in cortical network activation. For example, different aspects of semantic activity and language comprehension could be coded by different neural generators during the same time period, but go unnoticed when inspecting the N400 scalp distribution (Nobre and McCarthy, 1995; Lau et al., 2008). It is clear that spatial resolution is equally important for obtaining a complete picture of the neural activations underlying the semantic processes. However, by using high density EEG and source localization, one can reconcile the temporal and spatial resolution requirements needed to uncover the spatio-temporal dynamics of neural activity with an accuracy in the range of millimeters and milliseconds. We have adopted this technique to investigate the contribution of lexico-semantic features of word processing (orthographic/phonological, lexical access and semantic processing) and their spatio-temporal distribution in the brain. We hypothesize that, by performing statistical analysis directly on the sources as opposed to scalp-level analysis, finegrained differences previously obscured in EEG studies due to volume conduction can be revealed. In order to identify patterns of cortical activation we caution against scalp-level analysis, since many effects might be overlooked, as shown in this paper. To evaluate our paradigm, which calls upon the said semantic features, we developed a mass-univariate analysis approach on source level to discriminate between the different patterns of cortical activation.

\section{MATERIALS AND METHODS \\ Participants}

Twenty-two paid, healthy, native Dutch-speaking volunteers ( 9 males, 3 left-handed, average age $22 \pm 4$ years) participated in the study. The study was approved by the UZ Leuven ethics committee and conducted according to the latest version of the Declaration of Helsinki. All recruited subjects were first informed about the goal of the experiment, what would be expected from them, and what would be done with the collected data (privacy), after which they were invited to read and sign the informed consent form. No participant reported any history of neurological or psychiatric disorders. All participants had normal or corrected to normal vision.

\section{Word Stimuli}

We used 159 Dutch nouns combined from various categories: emotions and other abstract concepts, shapes, tools, colors, vegetables, food, fish, insects, furniture, sport disciplines, birds, and mammals.

We use the following semantic dimensions to discern our categories: word abstractness, potency, valence, and arousal. For our analysis all nouns were grouped based on these dimensions (note that within a single trial during the experiment, words could belong to any of the aforementioned semantic dimensions), and these dimensions were based on the three Osgood ratings (Moors et al., 2013) as well as on the concreteness ratings of Dutch words (Brysbaert et al., 2014). The words were chosen to have a score below 3.5 or above 4.5 for both poles of each dimension of the Osgood ratings, so as to ensure a statistically significant difference between the two groups on the same dimension (e.g., weak vs. strong for potency). The same principle applied to the abstractness ratings, abstract words should have a concreteness score below 2.5 and concrete words above 3.5. A $t$ test analysis showed a statistically significant difference between the two groups of each semantic dimension and the abstract and concrete groups based on their concreteness rating $(p<0.05$, high valence: $m=4.98$, std $=0.50$, low valence: $m=2.66$, std $=0.52$, high arousal: $m=5.13$, std $=0.40$, low arousal: $m=3.18$, std $=0.23$, high potency: $m=5.07$, std $=0.37$, low potency: $m=3.10$, std $=0.24$, abstract: $m=1.89$, std $=0.43$, concrete: $m=4.77$, std $=0.28$ ). These semantic categories were then further controlled along each dimension for word frequency, orthographic neighborhood size and word length (i.e., number of letters in a word) ratings taken from the Dutch CLEARPOND software (Marian et al., 2012): repeated measure ANOVA showed no significant difference between dimensions for word frequency and orthographic neighborhood size (for all dimensions, $p>0.05$ ). In the case of number of letters, no significant difference was between dimension potency and 
valence $(p>0.05)$, while differences in word length between abstract and concrete words and high and low arousal words were significant $(p=0.0043$ for abstractness and $p=0.0027$ for arousal). For these two word dimensions, for which we were not able to entirely control for word length, we repeated the same analysis by also including word length as a random factor. This analysis did not result in an elimination of any of the previous outcomes; however, in one case, these dimensions yielded additional activity regions (cf. discussion section). The entire dataset can be found in the Supplementary Material in Appendix A.

\section{Experimental Paradigm and Set-Up}

Participants were tested in a sound-attenuated and darkened room with a constant temperature of 20 degrees Celsius, sitting in front of an LCD screen at a distance of about $70 \mathrm{~cm}$. EEG data was recorded using 128 active $\mathrm{Ag} / \mathrm{AgCl}$ electrodes (SynampsRT, Compumedics, France) according to the international 10-5 system. Two of these electrodes served as ground (AFz) and reference $(\mathrm{FCz})$. The EEG signal was recorded at a $2 \mathrm{KHz}$ sampling rate and down sampled to $500 \mathrm{~Hz}$. All electrodes were mounted in an electrode cap, placed on the subject's head, for which we established the position of electrode $\mathrm{Cz}$ as the central point between inion, nasion, and the two pre-auricular points (Easycap, Germany). The cap was later used to obtain the electrode positions as pre-specified in the Brainstorm toolbox for source reconstruction. Conductive gel was applied in each of the electrode holes to ensure contact with the scalp.

During the experiment, in each trial, four white words on a black background were shown consecutively for $500 \mathrm{~ms}$ each, separated by a black screen for $1.2 \mathrm{~s}$ (inter-stimulus interval) with a jitter of $\pm 200 \mathrm{~ms}$. Three of these words were members of the same semantic category and the remaining word (called "filler") could either be a member of the same or a different semantic category. The order of this filler in the 4-word trial was randomized. Examples of such trials are shown in Table 1. We will refer to the remaining 3 words in the trial as "target words" since they will be analyzed and the filler word discarded.

Each trial started with a fixation cross that would cue the subject to gaze at the middle of the screen. After that, words were displayed and a cue was shown to prompt the subject to press the left mouse button if they thought all four words came from the same category (homogeneous trials) or the right button if otherwise (non-homogeneous trials). This semantic category matching task was chosen in order to ensure the proper depth of word-processing in our subjects (semantic processing, instead of mere lexical access, as would be expected for a lexical decision task). Note that the identification of these categories was not the goal of our study but rather used as a task for the subject. Subjects were asked not to click the button before the cue appeared in order to prevent contamination of our ERPs-of-interest with motor-related ones. After pressing a mouse button, they received visual feedback, which did not reflect the correctness of the trial but rather served as a reminder for the function of each mouse button ("goed!" (correct!) for the left button press, and "fout!" (wrong!) for the right button press).
The order of the trials was pseudo-randomized in a way that the same semantic category would not appear in two consecutive trials. Every subject repeated the experiment twice, with a break of about 20 min between the two sessions during which they performed another experiment so as to mitigate repetition effects. In total, the experiment lasted 30-40 min. The stimuli were presented using Matlab's Psychophysics Toolbox (Brainard, 1997).

\section{EEG Signal Pre-processing}

EEG data was re-referenced offline from the original ground and reference electrodes to a linked mastoid one and filtered using a 4th order Butterworth filter in the range of $0.1-30 \mathrm{~Hz}$. Even though the choice of the reference does not affect the inverse localization of neural active sources (Geselowitz, 1998; Pascual-marqui, 2007), we chose the linked mastoid reference because it was shown to yield a clearer N400 scalp response, which is one of the main ERP components used in the study of semantics (Marí-beffa et al., 2007; Kutas and Federmeier, 2011). For this reason, the linked-mastoid reference is the most commonly used one in linguistic ERP studies (Marí-beffa et al., 2007). The data was epoched using a window starting $100 \mathrm{~ms}$ prior to the presentation of the stimulus of interest (each of the 3 target words) until 1,000 ms post-onset. The average amplitude of $100 \mathrm{~ms}$ pre-stimulus signal was used to remove the poststimulus EEG signal baseline. Bad channels were eliminated for each subject based on a visual inspection of the data (an average of 18 channels were eliminated per subject), After this, trials in which the EEG amplitude on any of the electrodes exceeded $\pm 150 \mu \mathrm{V}$ were excluded as they could be due to motion artifacts.

\section{Source Localization}

For our source reconstruction analysis we used the Brainstorm toolbox (Tadel et al., 2011), freely available under the GNU general public license. The default anatomy was based on the ICBM-152 template. For the forward model we used OpenMEEG BEM (Gramfort et al., 2010), in which case the cortex was divided into 15,000 dipoles. The noise covariance matrix was obtained by merging the matrices calculated from the baseline of all selected trials. As our inverse modeling method, we used minimum norm estimates to estimate the sources and sLORETA (Pascual-Marqui, 2002) to normalize the estimated source density, as it has shown to yield zero localization errors in the absence of noise and to support the reconstruction of multiple sources. The source density is normalized at each point by a function of the data covariance and is unitless. Source orientation was constrained to be orthogonal to the cortical surface. The signal-to-noise ratio (SNR) was set to the default value suggested by the Brainstorm Toolbox (SNR = 3). In addition, sulci are not taken into consideration during our analysis, as accurate source localization in these regions is implausible, as stipulated in Brainstorm's documentation.

In order to verify the correctness of our procedure, we attempted to reproduce the results using different source localization algorithms, as is recommended by Mahjoory et al. (2017). We did not pursue the entire statistical procedure; 
TABLE 1 | Example trials.

\begin{tabular}{|c|c|c|c|c|}
\hline Trial type & word 1 & word 2 & word 3 & word 4 \\
\hline \multirow[t]{4}{*}{ homogeneous trial } & ansjovis (anchovy) & kabeljauw (codfish) & inktvis (squid) & snoek (pike) \\
\hline & knoflook (garlic) & ui (onion) & asperge (asparagus) & selderij (celery) \\
\hline & tang (pliers) & zaag (saw) & bijl (ax) & schop (shovel) \\
\hline & libel (dragonfly) & vlieg (fly) & mier (ant) & wesp (wasp) \\
\hline \multirow[t]{2}{*}{ non-homogeneous trial } & adelaar (eagle) & kanarie (canary) & specht (woodpecker) & kokosnoot (coconut) \\
\hline & jaloezie (jealousy) & vreugde (joy) & afkeer (aversion) & acteur (actor) \\
\hline
\end{tabular}

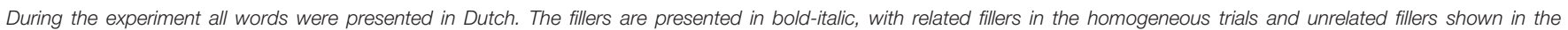
non-homogeneous trials. The three remaining words are the target words. English translations (between brackets) are for illustrative purposes only.

however, we did analyze our initial results by taking the average over all trials regardless of semantic features, using the four methods available in the brainstorm toolbox: wMNE, dSPM, sLORETA, and unconstrained sLORETA. Manual inspection of the results with these methods revealed that the spatial distributions are similar over time albeit with different degrees of spatial smoothing.

\section{Statistical Analysis}

For both scalp and source domain data, a mass-univariate approach using a linear mixed effect model was adopted (Verbeke and Molenberghs, 2000) with subjects and word length taken as random effect and semantic feature labels as fixed effects. In the case of the Osgood dimensions, we also corrected for the concreteness of the word by including it as a random effect. The dependent variable in the scalp domain was EEG amplitude and in the source domain it was dipole amplitude. Both averaged over $50 \mathrm{~ms}$ time bins between 0 and $900 \mathrm{~ms}$. The final model can be seen in Equation 1:

$$
\begin{aligned}
& \text { 'averaged amplitude' } \sim \text { semantic feature }+(1 \mid \text { Concreteness }) \\
& +(1 \mid \text { Subjects })+(1 \mid \text { WordLength })
\end{aligned}
$$

for the Osgood dimensions, and

$$
\begin{aligned}
& \text { 'averaged amplitude' } \sim \text { Concreteness }+(1 \mid \text { Subjects }) \\
& +(1 \mid \text { WordLength })
\end{aligned}
$$

for abstract vs. concrete words. In the source domain, averaging was performed for each of the 15,000 dipoles. Test results with $p$-values below 0.05 were considered significant. In cases such as ours, where dipoles are not independent from each other, and the number of dipoles is extremely large, Bonferroni- or FDR corrections are not appropriate as they will eliminate true positives (Cohen, 2014). Instead, we corrected for multiple comparisons using cluster-based inference adapted from the random field theory (Friston et al., 1994); as a threshold we took $3 \mathrm{~cm}^{2}$ of cortical surface as the minimal cluster size. The reasoning behind this threshold was based on a simulation study of the localization accuracy and is explained below. The same principle was applied for correcting over time, e.g., we only took those regions into consideration that were statistically significant for at least two consecutive time bins. When the measurement window and electrode sites are not known a priori, as in our case, one could rely on permutation tests to solve the multiple comparison problem in time and space simultaneously (Luck and Gaspelin, 2017). However, we felt the linear mixed effect to be a more appropriate model since it can account not only for inter-subject variability but also variability of any additional parameters (such as word length) and, therefore, has more power to detect small differences. Most importantly, the model allows us to control for the concreteness of the word when evaluating the Osgood dimensions, which is an important feature for our claim to suggest the dimensions as a way to ground both concrete and abstract words in the same underlying neural systems. Additionally, as suggested in many studies (Feise, 2002), we looked at effect size, specifically Cohen's d-effect size. Note that, as is the case with EEG studies, especially single word comprehension studies, differences are likely to be small and effect sizes of $0.01 \sim 0.1$ are to be expected (Kutas, 1993). In the scalp domain we also used cluster-based inference over electrodes by taking a cluster size of 10 adjacent electrodes, and only accepted clusters that were significant for at least 2 consecutive time bins, which would mean effects that last about $100 \mathrm{~ms}$, which corresponds to the approximate duration of transient EEG events (Koenig et al., 2002). It is very important to note that, since we performed the statistical analysis on relative values of dipole amplitudes (we do not rectify the signal for the mixed effect model), our results measured the distance between two conditions, showing the correct polarity of the signal. This is the case since the sign of the dipole amplitudes depends on the orientation of the source. However, the sign is ambiguous and cannot be used to claim that the brain response is stronger in one condition compared to another. Once our results are acquired, when studying a particular region of interest, rectified signals can be used to know which condition generates a larger response. This is possible since || $\mathrm{A}|-| \mathrm{B}|| \leq|\mathrm{A}-\mathrm{B}|$, which implies that the effect of rectified signals will always be smaller, therefore there is no need to perform statistical analysis on the rectified signals separately. Further information can be found in the tutorials on difference estimation provided by Brainstorm (http://neuroimage.usc.edu/brainstorm/ 


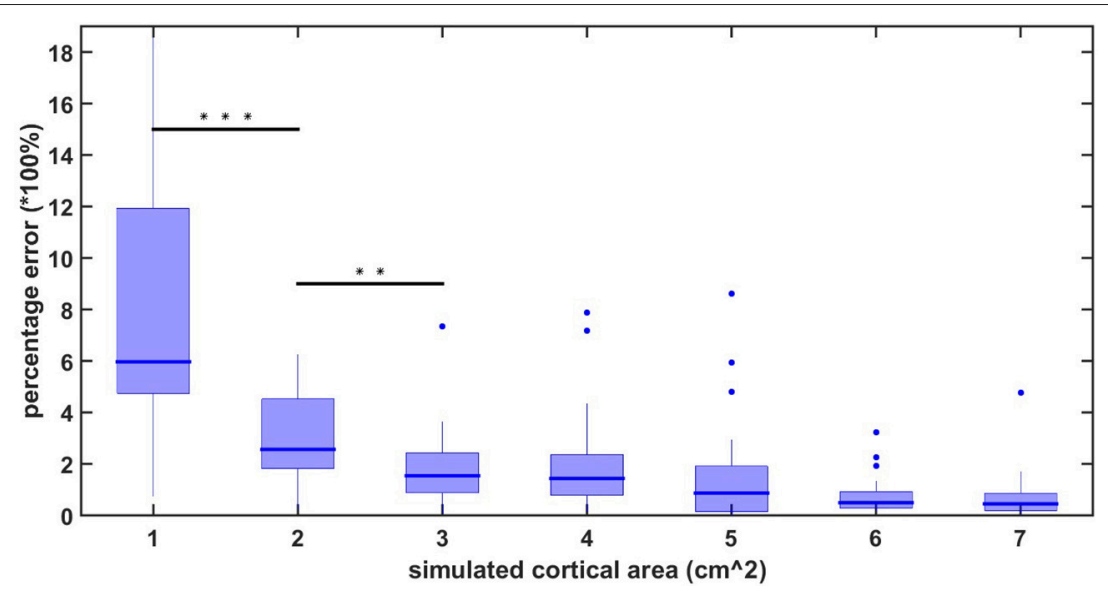

FIGURE 1 | localization percentage error for simulated source activity.

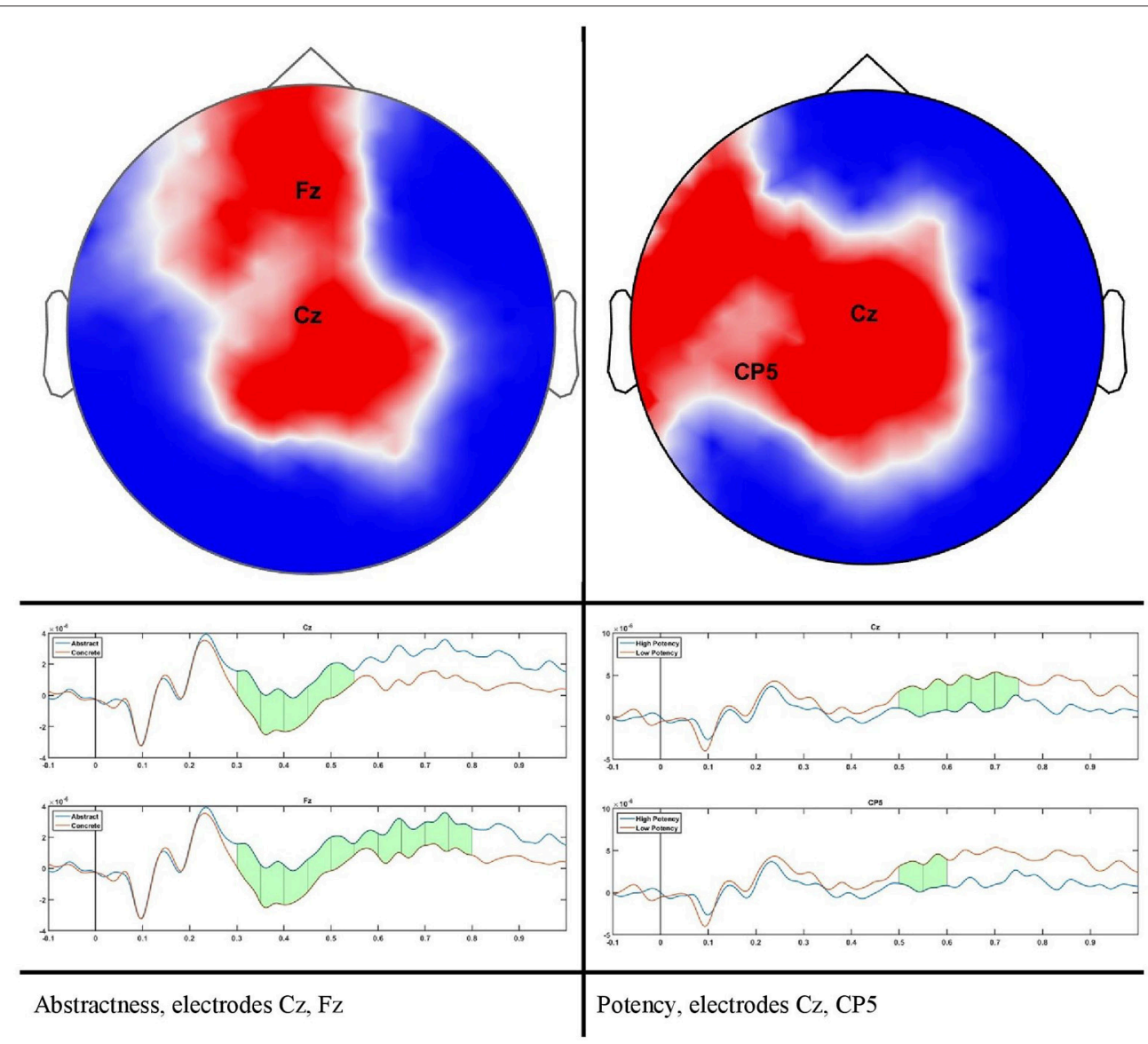

FIGURE 2 | scalp plot for significant differences and time course for two sample electrodes as indicated in scalp plots.

Tutorials/Difference). In addition to the aforementioned, we did not take into account regions that were located on the bottom of the sulci or in the interhemispheric fissure (we did take into account the wall of the gyri, but eliminated results at the bottom of the sulci as defined by Brainstorm), as source localization in these regions is suggested to be implausible and difficult to detect from EEG-based source localization.

The average localization error using the high-density EEG method applied in this study has previously been reported to be 


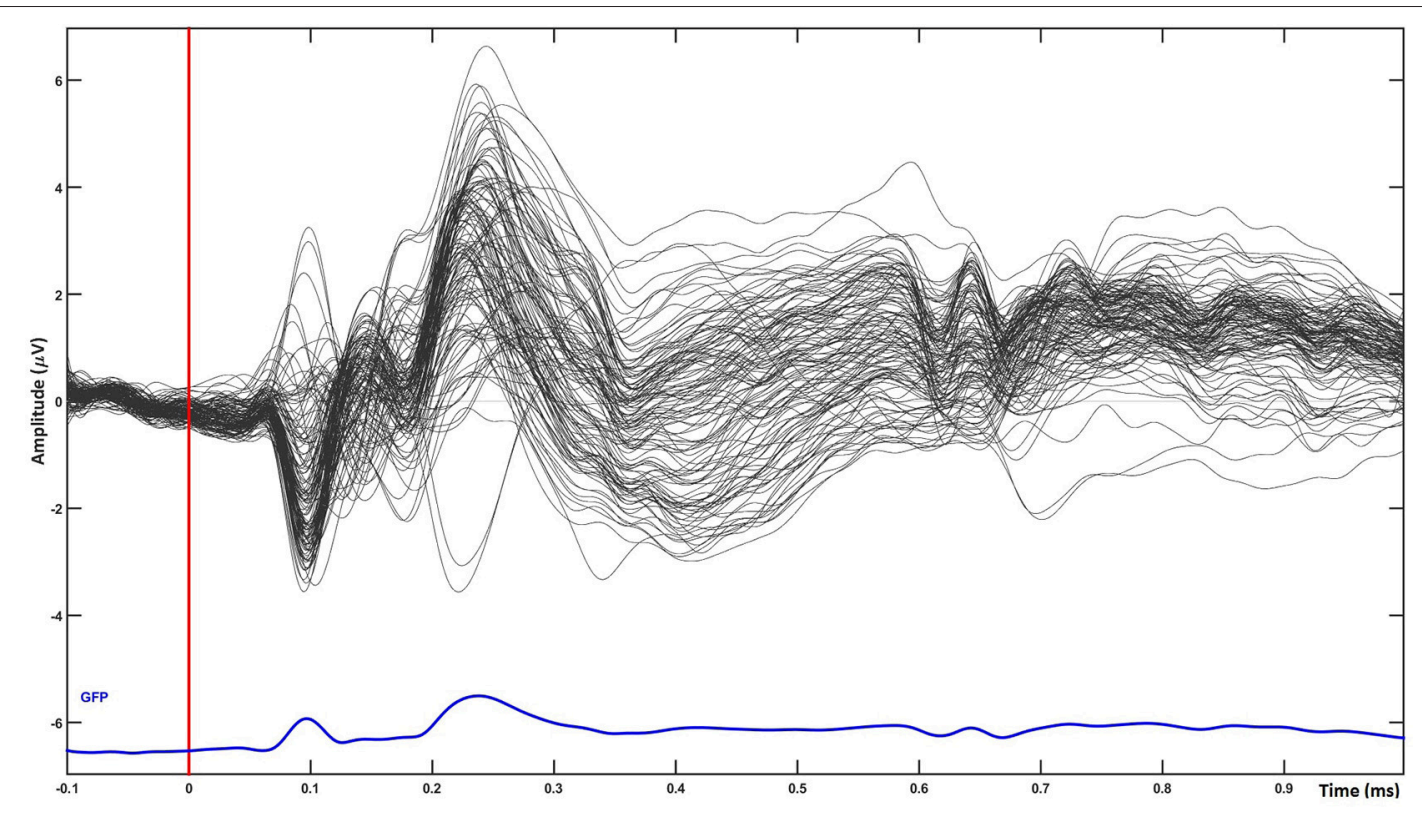

FIGURE 3 | temporal plot for single word processing averaged over all groups of words (grand average of all trials). The plot shows the quality of ERP data used for source analysis.

$10.5 \mathrm{~mm}$ for realistically shaped head models (Cuffin et al., 2001). However, these results are still under dispute (Song et al., 2015). Therefore, to ensure that the results of our statistical test are not merely localization inaccuracies, we performed a simulation study to measure the amount of crosstalk between sourcelocalized areas as a function of the spreading of activation. We simulated a signal consisting of a linear combination of sinusoids with frequencies ranging from 10 to $100 \mathrm{~Hz}$. We applied this signal to randomly chosen regions on the cortical surface with different square sizes. We chose 20 random areas for each simulated size of 1,2 , up to $7 \mathrm{~cm}^{2}$ of cortical surface. For each of these simulated regions, we applied the forward model to obtain simulated EEG signals on the scalp, and then applied the inverse solution to obtain an estimate of the spread of activity on the cortical surface. Since the activation of methods that employ L2 regularization, such as sLORETA's, will exhibit nonzero activity at all-time points and spatial locations (Gramfort et al., 2012), we defined the spreading of activation as the area outside which the amplitude of the dipoles falls below $50 \%$ of the maximum amplitude. The area of activity after determining the forward and inverse solutions was compared with the initially simulated activity in order to obtain a measure of the crosstalk (or spatial accuracy of the model). Figure 1 shows the percentage error (calculated as $\frac{\text { abs(calculated cortical area-simulated cortical area) }}{\text { simulated cortical area }}$ ) between the initially simulated and calculated area for surface region sizes between 1 and $7 \mathrm{~cm}^{2}$. As one can observe, the statistical difference between the region sizes drops below significance after $3 \mathrm{~cm}^{2}$ of simulated activity. Our cross-talk simulations confirmed that, for cortical surface activity above $3 \mathrm{~cm}^{2}$, the extent of spatial spreading does not differ significantly between any two cortical areas larger than $3 \mathrm{~cm}^{2}$. Therefore, in our linear mixed effect model on the cortical surface, we only retained areas of significance that where larger than $3 \mathrm{~cm}^{2}$.

\section{RESULTS}

\section{Scalp Analysis}

The scalp results obtained from the statistical analysis described previously are shown in Figure 2. A grand average of all trials shown in Figure 3 confirms the quality of our ERP data.

For abstractness, significant differences can be seen during the time course of $300-500 \mathrm{~ms}$ on a cluster of 23-28 channels $(P=0.0106$, Cohen's $d=0.042)$, and for potency during 500700 with a cluster size of $21-24$ electrodes $(P=0.0383$, Cohen's $d=0.0642$ ). No results remained after correcting for multiple comparisons for the dimensions of arousal and valence. This does not mean that no differences could be found on scalp level, but rather that our threshold for the cluster size is conservative.

\section{Source Analysis}

In order to evaluate any theory on word processing, a spatiotemporal view of brain activity is needed. When a single word is processed, categorized, and stored in semantic memory, a distributed network of cortical activity will emerge, starting to spread from the occipital areas toward the anterior temporal lobe (Borghesani, 2017). The entire activation network has been proposed to involve the left temporoparietal, angular, inferior and middle temporal gyri. When subjects are required to relate word meanings then several regions of the frontal lobe are additionally activated (Silva-pereyra et al., 2003).

When averaging across all word groups, source-localized brain activity can be seen (Figure 4) at different stages of 
$\sim 100 \mathrm{~ms}$

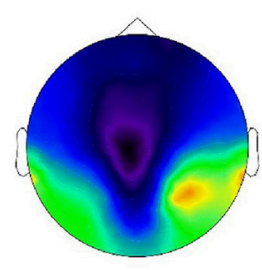

200ms

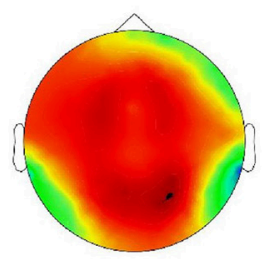

300ms

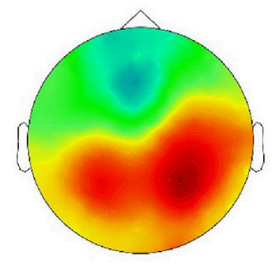

$\sim 400 \mathrm{~ms}$

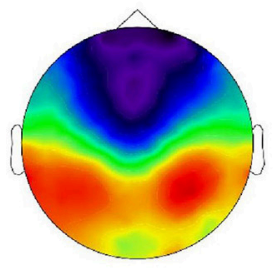

$\sim 500 \mathrm{~ms}$

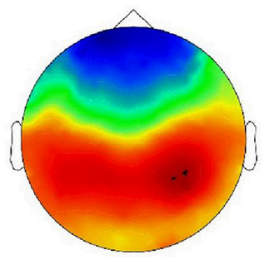

$\sim 600 \mathrm{~ms}$

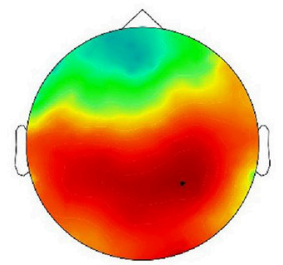

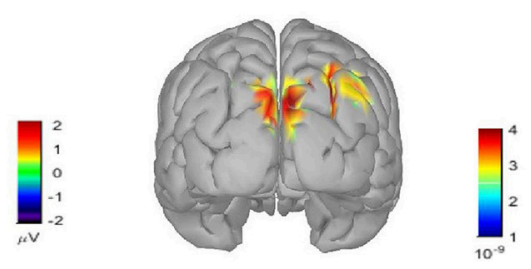
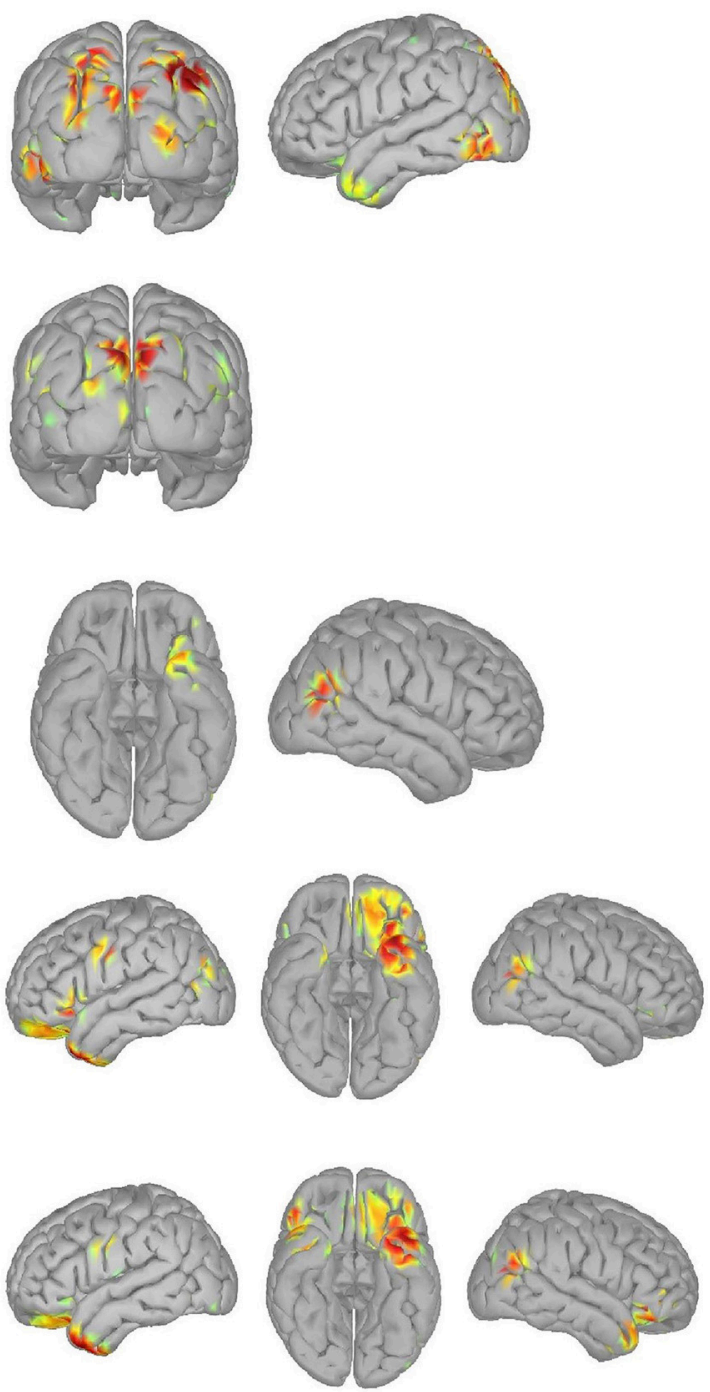

FIGURE 4 | scalp plot of time course of single word processing averaged over all groups, with associated source localization maps.

lexico-semantic processing. The early stages start with visual processing in the occipital lobe, then areas are recruited along the ventral stream, specifically the posterior occipital area of the left hemisphere around $200 \mathrm{~ms}$, until the posterior temporal lobe is reached in the time window of semantic processing $(400 \mathrm{~ms})$, and onwards anterior temporal, inferior frontal and orbital gyrus are included at later stages. This stream of activation has been previously shown with source localization studies (Borghesani, 2017). Therefore, together with the fact that our trials have only restricted variability in terms of noise as can be seen in Figure 3, this confirms the quality of source localization entering our statistical analysis.

\section{Results of Statistical Analysis on Source \\ Data}

Abstract vs. Concrete 
TABLE 2 | Statistical results for significance map of Figure 5 (abstractness).

\begin{tabular}{|c|c|c|c|c|c|c|c|c|c|}
\hline Region & $\begin{array}{l}\text { Time window } \\
\text { (ms) }\end{array}$ & $\begin{array}{c}\text { P value } \\
\text { (corrected) }\end{array}$ & Polarity & $\begin{array}{l}\text { Stronger } \\
\text { condition }\end{array}$ & Cohen's d & Size $\left(\mathrm{cm}^{2}\right)$ & $\begin{array}{l}\text { Estimate } \\
\left(10^{-10}\right)\end{array}$ & $\begin{array}{r}\text { Confi } \\
\text { interval }\end{array}$ & nce \\
\hline 1 right posterior MTG & $100-200$ & 0.013 & $A>C$ & $|A|>|C|$ & 0.040 & 4.42 & 5.31 & {$[2.86$} & 23.70] \\
\hline 2 right medial orbital gyrus & $450-650$ & 0.020 & $A>C$ & $|\mathrm{~A}|<|\mathrm{C}|$ & 0.025 & 22.96 & 10.80 & {$[3.18$} & 39.17] \\
\hline $\begin{array}{l}3 \text { right anterior inferior } \\
\text { temporal gyrus and MTG }\end{array}$ & $350-650$ & 0.023 & $A>C$ & $|A|>|C|$ & 0.036 & 24.18 & 15.86 & {$[4.63$} & 66.84] \\
\hline 4 left anterior MTG and ITG & $300-550$ & 0.029 & $A>C$ & $|A|>|C|$ & 0.032 & 37.04 & 14.96 & {$[1.02$} & 59.71] \\
\hline 5 left STG/STS* & $250-700$ & 0.015 & $A>C$ & $|\mathrm{~A}|>|\mathrm{C}|$ & 0.041 & 12.71 & 7.72 & {$[4.68$} & 34.95] \\
\hline 6 left superior parietal gyrus & $700-1,000$ & 0.018 & $A>C$ & $|A|<|C|$ & 0.038 & 10.25 & 6.74 & {$[2.89$} & 29.33] \\
\hline 7 right inferior parietal gyrus & $0-100$ & 0.018 & $A<C$ & $|A|<|C|$ & 0.040 & 4.68 & 2.87 & {$[-12.77$} & $-1.50]$ \\
\hline 8 right postcentral gyrus & $0-100$ & 0.010 & $\mathrm{~A}<\mathrm{C}$ & $|A|>|C|$ & 0.045 & 9.01 & 2.33 & {$[-11.16$} & $-2.00]$ \\
\hline 9 left MTG/MTS* & $300-700$ & 0.018 & $\mathrm{~A}<\mathrm{C}$ & $|A|>|C|$ & 0.039 & 14.17 & 9.33 & {$[-40.83$} & $-4.22]$ \\
\hline 10 left middle parietal gyrus & $700-1,000$ & 0.019 & $\mathrm{~A}<\mathrm{C}$ & $|A|<|C|$ & 0.036 & 9.91 & 7.47 & {$[-31.53$} & $-2.22]$ \\
\hline
\end{tabular}

A, Abstract; C: Concrete.

* The activation is located on the wall of sulcus but does not include the bottom.

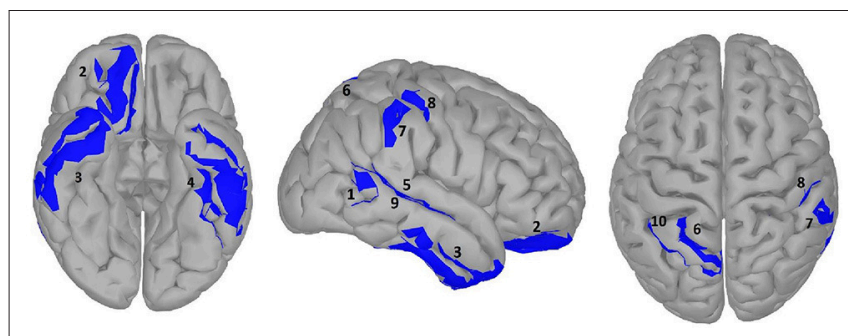

FIGURE 5 | significance map for abstractness.

\section{Arousal}

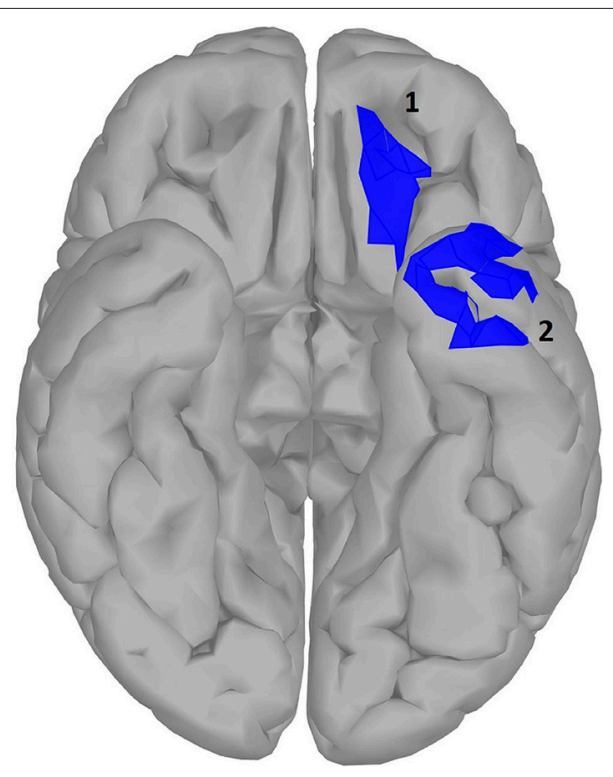

FIGURE 6 | significance map for arousal.

\section{Potency}

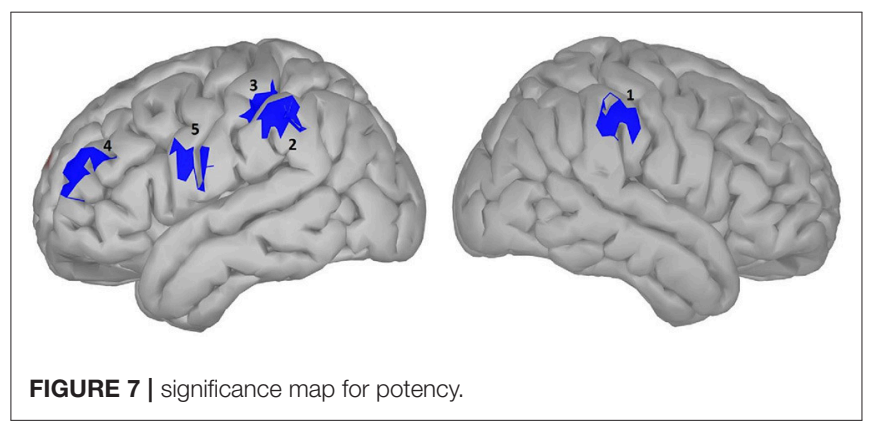

Valence

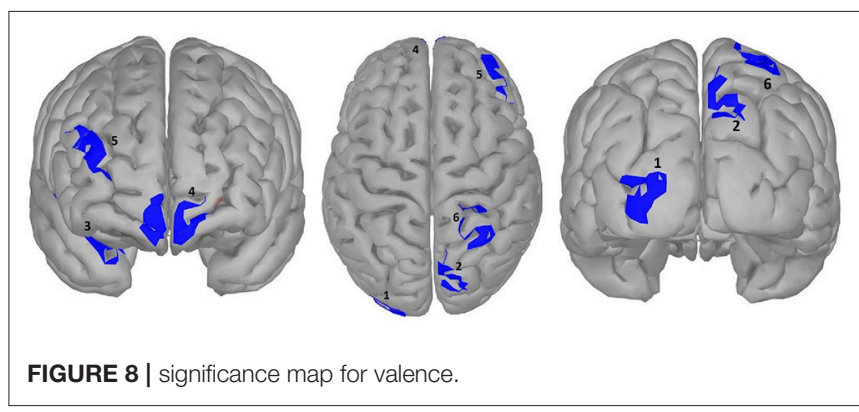

\section{DISCUSSION}

\section{Effect of Abstractness: Abstract vs.} Concrete Words

A large difference was observed in the scalp domain during the time window of semantic processing, located centro-parietally 


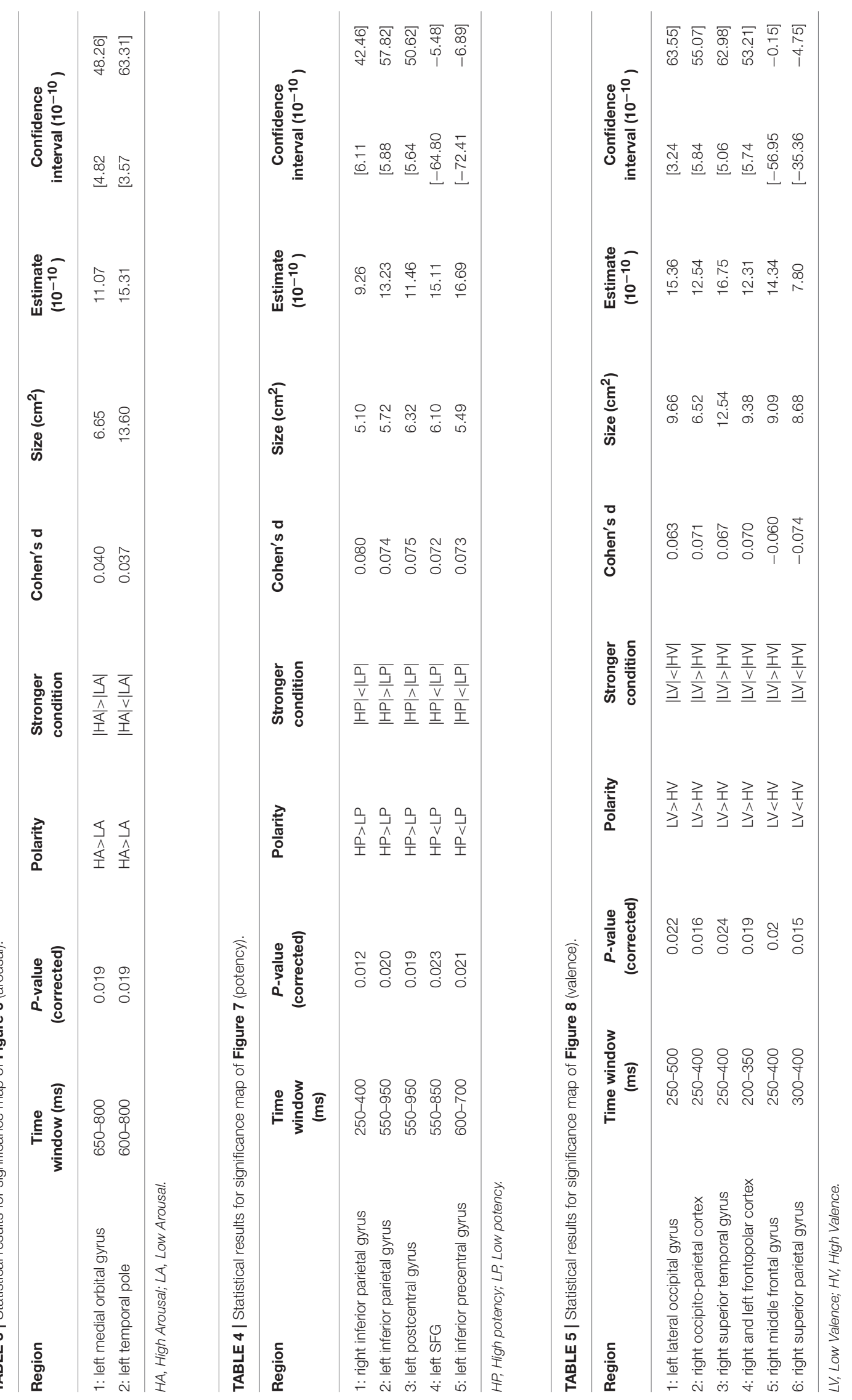


(Figure 2, 300-500 ms), in agreement with previous reports on the effect of abstractness in word processing (West and Holcomb, 2000; Kanske and Kotz, 2007). This result translated to a few regions in the source analysis (Figure 5: regions 2 and 3, and Table 2). Significant differences in sources between abstract and concrete words are present very early on in the posterior parietal areas (the time-interval for visual and orthographic processing) followed by the left and right temporal cortex and the right inferior frontal cortex (time-interval of semantic processing), and the occipito-parietal areas toward the end of the $900 \mathrm{~ms}$ time-window. Regions located in the left hemisphere such as the fusiform and middle temporal gyrus are akin to those found with fMRI studies (Wang et al., 2013). Additionally, a large involvement of the right temporal cortex in processing abstract words, especially during the time interval of semantic retrieval, suggests the involvement of more brain areas in the processing of abstract concepts in the right hemisphere. This is similar to the work of Kiehl et al. (1999), who suggested a right hemisphere neural pathway for processing abstract words. For most brain areas that were, according to Perani et al. (1999), involved in processing abstractness, we observed larger activity in response to abstract compared to concrete words. However, unlike their study, where activity was observed only in the left posterior temporal and bilateral frontal cortices, we observed additional activity in the right temporal cortex and the occipito-parietal cortices in the right hemisphere, consistent with some PET studies suggesting an interpretative role of the right hemisphere in processing abstract language (Beauregard et al., 1997; D’Esposito et al., 1997). A possible reason for why our study revealed more areas in the right hemisphere, compared to similar fMRI studies such as Kiehl's (Kiehl et al., 1999), could be that with EEG, given its excellent temporal resolution, short-lasting activations are more visible than with fMRI.

Another important fact to mention is the time window of semantic processing, usually linked to a scalp ERP component referred to as the $\mathrm{N} 400$, a negative amplitude deflection that peaks around $300-500 \mathrm{~ms}$ in response to a potentially meaningful stimulus (Lau et al., 2008; Kutas and Federmeier, 2011). Most studies observe a larger N400 amplitude for concrete words than for abstract ones (Kounios and Holcomb, 1994), as is also the case with our study (Figure 2). However, there is no consensus on the location of this difference as some suggest it to be in the right hemisphere (Kounios and Holcomb, 1994) while others suggest the opposite (Kiehl et al., 1999). Kiehl argues that this apparent contradiction could be mainly due to a different choice in electrode referencing. We suggest to take this one step further by arguing that, in addition to the choice of the electrode reference, the lateral direction of scalp activity is not always an indication of the underlying source as, depending on the orientation of dipole activity, scalp activity can originate from a region that is not directly beneath it (Gloor, 1985). However, this issue can be resolved when using source reconstruction as the location of the neural sources is reference-independent (Michel et al., 2004). Therefore, we claim our results to be more objective than those of previous scalp ERP studies and, in this way, we can conclude that the right hemisphere plays a bigger role in the difference between brain activation in response to abstract and concrete words.

\section{Effect of Arousal: Active vs. Passive Words}

Arousal and valence have been suggested to be part of a twodimensional model of emotional word processing (Citron, 2012; Citron et al., 2013). In this framework, some studies have questioned whether these dimensions are actually distinct or correlated (Bradley and Lang, 1999). However, in our study no correlation was found between the Osgood ratings of arousal and valence in our word dataset. In the temporal domain, we did not observe any difference large enough to outlive correction between high and low arousal words. Similar EEG studies, using a lexical decision task to investigate the effect of arousal in single word processing, did observe ERPs in early time windows to be modulated by arousal (Hofmann et al., 2009; Conrad et al., 2014) but in these studies stimuli are controlled for valence (words were all negative), which was not the case with our study. Additionally in these studies, window selection was less conservative compared to ours. When using alternative modalities such as images of brand logos instead of written stimuli, again no effect of arousal was observed (Schaefer and Rotte, 2010). Other studies (Lewis et al., 2007; Aryani et al., 2018) observed effects of increasing arousal in deep structures such as the amygdala and putamen, which is very difficult to observe with EEG (see discussion). However, in our source localization analysis (results shown in Figure 6 and Table 3), we did observe two localized areas in the left hemisphere similar to those seen by Aryani et al. (2018), temporal pole and medial orbital gyrus, that were modulated by word arousal. These areas resemble the left superior and middle temporal gyrus and the middle frontal gyrus reported by Kawachi et al. (2011), which was closest to ours although it was done with fMRI. Differences for both these regions occur relatively late in word processing, around 650 $800 \mathrm{~ms}$. The late effect can be explained by the claim that during the integration process of a word in working memory (600$800 \mathrm{~ms}$ ), words with high arousal require more activation from the brain area involved in word integration (Lau et al., 2008), which can be seen only in the medial orbital gyrus. On the other hand, the left temporal pole, including the anterior superior temporal gyrus, shows increased activity for passive words. Note that in this study, the parietal gyrus was also mentioned to exhibit more activity, a plausible result since the superior parietal cortex is suggested to be involved in attention allocation (Ptak, 2012; Gonzalez et al., 2015). Given this, we can assume that for the initial processing of active words less attention is needed and therefore their retrieval easier. It is interesting to mention that when we took word length as an additional random variable into account in our model, the superior parietal cortex indeed showed significantly higher activation for passive words in the early time windows (100-200 ms, results not shown).

\section{Effect of Potency: Strong vs. Weak Words}

We did not observe any effect of potency during the time window of semantic processing in either the scalp or the source domain, but in a later window an effect was observed, mainly a smaller amplitude for high potency stimuli compared to low ones that were lateralized in the left hemisphere, specifically the inferior parietal and the superior frontal and inferior precentral gyrus, where only the inferior parietal lobe showed higher 
activation for stimuli with a high potency value. The inferior parietal region was mirrored in the right hemisphere, albeit in an earlier time-window than the left hemisphere, since it was activated in response to word potency during lexical access and semantic retrieval, while other areas were activated starting from a late time window $(550 \mathrm{~ms})$ and lasted until the late stages of word processing (around $800 \mathrm{~ms}$ ), (scalp domain results shown in Figure 2, source domain in Figure 7 and Table 4). The larger activation for low compared to high potency in the superior frontal cortex has been reported in several other studies, using both fMRI and EEG (Skrandies, 1998; Schaefer and Rotte, 2010; Kawachi et al., 2011). This could be because high potency words are processed easier (lexical access and semantic retrieval), as evidenced by physiological responses recorded during behavioral tests (Bradley et al., 2001), reducing the load of working memory and cognitive control and the involvement of the pre-frontal cortex (Macdonald et al., 2000). Considering these studies involve both written and visual stimuli, we believe this activation to be independent of modality. Studies involving this dimension have been generally scarce, since the majority of studies investigating the neural mechanism of emotional processing focus on Osgood's two other affective meaning dimensions, valence and activity (Fontaine et al., 2013). However, as our study shows, the potency dimension should not be overlooked.

\section{Effect of Valence: Negative vs. Positive Words}

The effect of valence in the temporal domain was significant starting from $200 \mathrm{~ms}$ until around $500 \mathrm{~ms}$ (results shown in Figure 8, and Table 5). This effect starts slightly earlier compared to some previous studies, where the effect was observed starting from 250 to $290 \mathrm{~ms}$ (Palazova et al., 2013; Imbir et al., 2016). This could be due to the used task, as in both cited studies a lexical decision task was adopted instead of a categorization task, as in our case, which calls upon semantic processing. One comprehensive overview of written emotion word processing using both fMRI and EEG (Citron, 2012) showed that, for the majority of the cited studies that adopted a lexical decision task, a time window of $200-300 \mathrm{~ms}$ marked the onset of the effect of emotional content of verbal material (i.e., a larger amplitude for emotionally charged words over neutral words). However, the same review also suggested a later time window of $500-800 \mathrm{~ms}$ which we did not observe. In our source localization analysis, we observed the early time window effect in the left lateral occipital cortex, the right occipitoparietal, middle frontal and superior temporal gyrus, and the prefrontal (frontopolar) cortex bihemispherically. The overall effect was therefore more lateralized in the right hemisphere, which is in line with the commonly accepted processing of emotion in the right hemisphere, including fMRI studies with similar paradigms (Kuchinke et al., 2005; Citron, 2012). In the occipital, prefrontal and occipito-parietal areas, similar to previous ERP studies (Kawachi et al., 2011; Palazova et al., 2013), words with high valence evoke a higher activation in early time windows compared to words with low valence. In the processing of valence, unlike other semantic features, the temporal cortex was involved to a considerably lesser degree and mainly only in the right hemisphere (anterior part of superior temporal gyrus). Activation in the middle frontal cortex was confirmed by similar fMRI studies (Schaefer and Rotte, 2010). On the other hand, also unlike other semantic features, the right superior parietal lobule was primarily involved in processing valence specifically during lexical access and semantic processing $(250-500 \mathrm{~ms})$. This could be explained by the involvement of the parietal cortex in processing emotionally salient stimuli (Barbaro et al., 2017). It is interesting to note that many studies have suggested valence as an embodied approach toward the neural representation of abstract concepts (Kousta et al., 2011; Vigliocco et al., 2014). Our study shows the same distinct neural patterns to represent both abstract and concrete concepts when correcting for levels of concreteness in the mixed model.

\section{GENERAL DISCUSSION}

In order to shed light on the possible neural correlates of semantic category representation, we sat out to chart the networks whose activity differs when processing different semantic features. When evaluating the processing of semantic features, mainly techniques with either good temporal (ERP) or spatial (fMRI) resolution have been adopted, thus addressing either the when or where questions. We believe that high-density EEG with source localization yields the best of both worlds as it combines spatial with temporal resolution. To this end, we have developed a massunivariate analysis technique in the source domain using a mixed linear effect model.

To summarize our results, a few regions seem to be playing an important role in processing semantic features. First, we identified the left inferior frontal lobe, which is in line with the increased fMRI activation observed in the lateral inferior prefrontal cortex during deep semantic encoding (Demb et al., 1995; Bookheimer, 2002), from which the authors argued that it might play a central executive role in retrieving semantic information. Indeed, the activity of the inferior frontal lobe seems to differ between different semantic features such as abstractness and activity. In agreement with clinical observations, early functional brain imaging studies of semantic processing revealed activity in broad regions of the left prefrontal, parietal and posterior temporal lobes, commonly including ventral and lateral regions of the temporal cortex (Martin and Chao, 2015). Studies have shown that the ventral and lateral regions of the posterior temporal cortex can be differently engaged in semantic processing, depending on the type of information retrieved. This is consistent with the results from our study, where the temporal cortex seems to be prominently active when processing various semantic features.

On the same note, we believe that the role of the right hemisphere in word processing should be more acknowledged since, in the current study, we found a number of brain areas, in 
particular in the right temporal cortex, that were largely involved in processing a number of semantic features (i.e., abstractness and valence). This activation was mainly present during lexical access and semantic processing (300-600 ms), providing evidence of the role of the right hemisphere in the semantic processing of a word, as previously reported using mono-hemispheric stimulation studies (Coulson et al., 2005). Finally, certain brain areas participate in the processing of several word features. For instance, the involvement of fronto-temporal areas in our study, similar to some previous investigations (Lau et al., 2008; Brouwer and Hoeks, 2013), was observed in the processing of most features-of-interest. However, it is worth noting that none of the listed areas participated in the processing of all features.

Processing abstract and concrete words involves partially overlapping brain networks. The larger engagement of the left hemisphere over the right when processing abstract words seems to be in favor of the dual coding theory (Binder et al., 2005), but overall we did not observe a consistently larger activation of the right hemisphere for concrete words and cannot support this theory exclusively. When considering the size of the differences, the total area with a stronger activation for abstract words was twice the amount in the opposite condition, and this ratio increased to quadruple the original amount when taking into account only the approximate time window for semantic processing (Table 4). These findings suggest a richer network of brain activation for processing abstract concepts. To the best of our knowledge, we are the first to demonstrate this, and we believe the richer activation can explain the behavioral effect of concreteness, since a larger network would imply more effort and therefore a longer response time for abstract concepts. Since abstract words depend on a looser set of associated knowledge than concrete words, previous research (Binder et al., 2005) has speculated this would transform into stronger activation for concrete words. However, stronger associated knowledge would imply less processing effort. Other studies have also suggested a more extensive spatial distribution in the absence of strong semantic context during single word processing (Wang et al., 2013). Therefore, we believe our result of stronger activation for abstract concepts to be in support of the context availability theory. In the end, our results do not entirely support one single theory over another but combine aspects of both.

The semantic dimensions defined by Osgood show a distributed network of differences. In the current study, dimensions of valence and potency had a higher effect size and occurred earlier in the processing chain compared to activity. This points in the direction of a higher biological value for valence and potency over activity, as their evaluation does not require language since we have evolved to react to more biologically significant stimuli to preserve our lives (Imbir et al., 2016). As such, these dimensions could indeed serve as the necessary semantic space to describe both abstract and concrete concepts. Though we did include concreteness as a random factor in our model, further study is necessary to investigate the interaction between concreteness and these dimensions also to establish whether (which part of) the distributed network is shared by the two dimensions. Additionally, some studies have questioned the distinction of the dimensions and have suggested that there is in fact a high correlation between arousal and valence ratings, which we did not find in our database. Other studies have reported that the self-assessment ratings of potency (as we used in our study) diverge from the original semantic differential scale suggested by Osgood since subjects are rating their own feeling of dominance rather than the dominant meaning of the word (Bradley and Lang, 1994). As for the Moors et al. database we used, the dominant meaning of the word was rated (Moors et al., 2013). Since the correlations between dimensions were also reported, we could verify them for our dataset. We found a weak but significant positive correlation between potency and arousal. To investigate the possible implications of such interactions between these dimensions would constitute a study on its own and is beyond the scope of the present study. Regardless, we do not believe the outcome would undermine our hypothesis, since our purpose was to unveil the spatio-temporal activation patterns for individual Osgood dimensions when processing abstract and concrete words.

On a final note, in EEG source reconstruction two mainstream techniques are used: the equivalent current dipole modeling (parametric) and distributed source models (nonparametric, Grech et al., 2008). The former requires prior specification of the number of neural sources and will solve the inverse solution to find the location and orientation for these sources. The latter assumes the entire cortical surface (or brain volume) to consist of fixed source locations and will solve the issue of their amplitude, but these sources are by no means assumed to be independent and can therefore not be regarded as different neural generators. Since we had no prior knowledge of where to expect our effect (as language processes generally involves a large area of activity; Price, 2015), we used distributed source analysis instead of equivalent current dipoles. However, since we did not perform any analysis to estimate how many sources are interacting, we do not claim that differences in source localization are due to different neural generators, for example both areas located in the inferior parietal lobe of the left hemisphere for potency are likely to originate from the same generator.

\section{LIMITATION AND FUTURE STUDY}

One limitation of our study was that the solution space for source localization was confined to the cortical surface. This prevented us from investigating activity from deeper structures. Several studies suggested that such activity could be detected from EEG source reconstruction (Attal et al., 2009; Cebolla et al., 2016). Admittedly, the results of our statistical analysis also included medial areas of the cerebral cortex such as the frontopolar gyrus and gyrus rectus, located in the frontal part of the interhemispheric fissure (mainly abstract words, see Supplementary Material in Appendix B, for detailed representation). However, since the contribution of these sources to EEG scalp activity is still under much debate (Attal et al., 2012), we did not include these results in the main manuscript. In addition, because our head model is confined to the cortical surface, it is possible that the activity we observe does in fact originate from subcortical regions, forcedly mapped onto the 
most nearby cortical surface. To understand where this activation truly comes from, future studies would be necessary to perform source localization on the entire cortical volume, including deeper structures such as the thalamus and cerebellum.

\section{CONCLUSION}

The purpose of our study was to demonstrate differences in source activation when processing abstract and concrete words also when scoring extremally along the valence, arousal, and potency semantic dimensions defined by Osgood. Our results show that each dimension has a distinct spatio-temporal pattern of activation for the low and high values of a given dimension when correcting for the concreteness of the word. These results are promising in that they could indeed provide evidence for grounding both abstract and concrete words in the same underlying neural system and, in this way, pave the way toward a unified theory on semantic category representation. Since each EEG electrode records a mix of activity from multiple sources (Nunez and Srinivasan, 2006), we believe that the un-mixing performed by source localization can reveal effects previously unnoticed. With the current study, we showed that different neural populations respond to different semantic dimensions. Here, we studied the temporal and spatial dynamics of processing the semantic features of a word. We did not consider words as plain linguistic entities, but rather as complex combinations of lexical and semantic characteristics. Given that our source localization technique allowed us to identify the brain areas involved in semantic feature processing during different stages of word processing, we are in a position to update or alter existing models of spatio-temporal dynamics of word processing.

\section{REFERENCES}

Aryani, A., Hsu, C. T., and Jacobs, A. M. (2018). The sound of words evokes affective brain responses. Brain Sci. 8:94. doi: 10.3390/brainsci8060094

Attal, Y., Bhattacharjee, M., Yelnik, J., Cottereau, B., Okada, Y., Lefevre, J., et al. (2009). Modelling and detecting deep brain activity with MEG and EEG. IRBM 30, 133-138. doi: 10.1016/j.irbm.2009.01.005

Attal, Y., Maess, B., Friederici, A., and David, O. (2012). Head models and dynamic causal modeling of subcortical activity using magnetoencephalographic/electroencephalographic data. Rev. Neurosci. 23, 85-95. doi: 10.1515/rns.2011.056

Barbaro, L., Peelen, M. V., and Hickey, C. (2017). Valence, not utility, underlies reward-driven prioritization in human vision. J. Neurosci. 1128-17. doi: 10.1523/JNEUROSCI.1128-17.2017

Barsalou, L. W. (2008). Grounded cognition. Annu. Rev. Psychol. 617-745. doi: 10.1146/annurev.psych.59.103006.093639

Barsalou, L. W. (2010). Grounded Cognition : Past, Present, and Future. Top. Cogn. Sci. 2, 716-724. doi: 10.1111/j.1756-8765.2010.01115.x

Barsalou, L. W., Simmons, W. K., Barbey, A. K., and Wilson, C. D. (2003). Grounding conceptual knowledge in modality-specific systems. Trends Cogn. Sci. 7, 84-91. doi: 10.1016/S1364-6613(02)00029-3

Beauregard, M., Chertkow, H., Bub, D., Murtha, S., Dixon, R., and Evans, A. (1997). The neural substrate for concrete, abstract, and emotional word lexica: a positron emission tomography study. J. Cogn. Neurosci. 9, 441-461. doi: 10.1162/jocn.1997.9.4.441

Bentin, S., Mouchetant-Rostaing, Y., Giard, M. H., Echallier, J. F., and Pernier, J. (1999). ERP manifestations of processing printed words at different

\section{AUTHOR CONTRIBUTIONS}

MF conceived and conducted the experiments and performed the analysis. EK, MF, and MMVH participated equally in writing and reviewing the manuscript.

\section{FUNDING}

MF is supported by the Hermes Fund of the National Fund for Scientific Research Flanders (SB/ 151022). EK is supported by the Belgian Fund for Scientific Research-Flanders (G088314N). $\mathrm{MMVH}$ is supported by research grants received from the Financing program (PFV/10/008), the interdisciplinary research fund (IDO/12/007) and the special research fund of the KU Leuven (C24/18/098), the Belgian Fund for Scientific Research-Flanders (G088314N, G0A0914N, G0A4118N), the Interuniversity Attraction Poles Programme-Belgian Science Policy (IUAP P7/11), the Flemish Regional Ministry of Education (Belgium) (GOA10/019), and the Hercules Foundation (AKUL 043).

\section{ACKNOWLEDGMENTS}

We would also like to thank Mathias Sjerps for proofreading the manuscript and giving his comments.

\section{SUPPLEMENTARY MATERIAL}

The Supplementary Material for this article can be found online at: https://www.frontiersin.org/articles/10.3389/fnhum. 2018.00503/full\#supplementary-material

psycholinguistic levels : time course and scalp distribution. J. Cogn. Neurosci. 11, 235-260. doi: 10.1162/089892999563373

Binder, J. R., Desai, R. H., Graves, W. W., and Conant, L. L. (2009). Where is the semantic system ? A critical review and meta-analysis of 120 functional neuroimaging studies. Cereb. Cortex 19, 2767-2796. doi: 10.1093/cercor/bhp055

Binder, J. R., Westbury, C. F., McKiernan, K. A., Possing, E. T., and Medler, D. A. (2005). Distinct brain systems for processing abstract and concrete concepts. J. Cogn. Neurosci. 17, 905-917. doi: 10.1162/0898929054021102

Bookheimer, S. (2002). FUNCTIONAL MRI OF LANGUAGE : new approaches to understanding the cortical organization of semantic processing. Annu. Rev. Neurosci. 25, 151-188. doi: 10.1146/annurev.neuro.25.112701.142946

Borghesani, V. (2017). The Neuro-Cognitive Representation of Word Meaning Resolved in Space and Time. Trento: University of Trento.

Bradley, M. M., Codispoti, M., Cuthbert, B. N., and Lang, P. J. (2001). Emotion and motivation I : defensive and appetitive reactions in picture processing. Emotion 1, 276-298. doi: 10.1037/1528-3542.1.3.276

Bradley, M. M., and Lang, P. J. (1994). Measuring emotion : the selfassessment semantic differential. J. Behav. Ther. Exp. Psychiatry 25, 49-59. doi: 10.1016/0005-7916(94)90063-9

Bradley, M. M., and Lang, P. J. (1999). Affective norms for English words (ANEW): instruction manual and affective ratings. Tech. Rep. C-1, Cent. Res. Psychophysiol. 30, 25-36.

Brainard, D. H. (1997). The psychophysics toolbox. Spat. Vis. 10, 433-436. doi: $10.1163 / 156856897 X 00357$

Brouwer, H., and Hoeks, J. C. (2013). A time and place for language comprehension: mapping the $\mathrm{N} 400$ and the P600 to a minimal cortical 
network. Front. Hum. Neurosci. 7:758. doi: 10.3389/fnhum.2013. 00758

Brysbaert, M., Stevens, M., De Deyne, S., Voorspoels, W., and Storms, G. (2014). Norms of age of acquisition and concreteness for 30, 000 Dutch words. Acta Psychol. 150, 80-84. doi: 10.1016/j.actpsy.2014.04.010

Caramazza, A., and Mahon, B. Z. (2003). The organization of conceptual knowledge : the evidence from category-specific semantic deficits. Trends Cogn. Sci. 7, 354-361. doi: 10.1016/S1364-6613(03)00159-1

Cebolla, A. M., Petieau, M., Dan, B., Balazs, L., McIntyre, J., and Cheron, G. (2016). Cerebellar contribution to visuo-attentional alpha rhythm: insights from weightlessness. Sci. Rep. 6, 37824. doi: 10.1038/srep37824

Citron, F. M. (2012). Neural correlates of written emotion word processing : a review of recent electrophysiological and hemodynamic neuroimaging studies. Brain Lang. 49, 1-49. doi: 10.1016/j.bandl.2011.12.007

Citron, F. M. M., Weekes, B. S., and Ferstl, E. C. (2013). Effects of valence and arousal on written word recognition : time course and ERP correlates. Neurosci. Lett. 533, 90-95. doi: 10.1016/j.neulet.2012.10.054

Cohen, M. X. (2014). "Advantages and limitations of different statistical procedures," in Analyzing Neural Time Series Data. (London, UK: MIT Press).

Conrad, M., Recio, G., Conrad, M., Hansen, L. B., and Jacobs, A. M. (2014). On pleasure and thrill : the interplay between arousal and valence during visual word recognition Brain \& Language On pleasure and thrill : the interplay between arousal and valence during visual word recognition. Brain Lang. 134, 34-43. doi: 10.1016/j.bandl.2014.03.009

Coulson, S., Federmeier, K. D., Van Petten, C., and Kutas, M. (2005). Right hemisphere sensitivity to word- and sentence-level context: evidence from event-related brain potentials. J. Exp. Psychol. Learn. Mem. Cogn. 31, 129-147. doi: 10.1037/0278-7393.31.1.129

Cuffin, B. N., Schomer, D. L., Ives, J. R., and Blume, H. (2001). Experimental tests of EEG source localization accuracy in realistically shaped head models. Clin. Neurophysiol. 112, 2288-2292. doi: 10.1016/S1388-2457(01)00669-1

Demb, J. B., Desmond, john, E., Wagner, A. D., Vaidya, C. J., and Glover, G. H., Gabrieli, J. D. (1995). Semantic encoding and retrieval in the left inferior prefrontal cortex : a functional MRI study of task difficulty and process specificity. J. Neurosci. 15, 5870-5878. doi: 10.1523/JNEUROSCI.15-09-05870.1995

D’Esposito, M., Detre, J. A., Aguirre, G. K., Stallcup, M., Alsop, D. C., Tippet, L. J. et al. (1997). A functional MRI study of mental image generation. Neuropsychologia 35, 725-730.

Feise, R. J. (2002). Do multiple outcome measures require p-value adjustment? BMC Med. Res. Methodol. 4:8. doi: 10.1186/1471-2288-2-8

Fontaine, J. J., Scherer, K. R., and Soriano, C. (2013). "Reviving a forgotten dimension-potency in affective neuroscience," in Components of Emotional Meaning: A Sourcebook. Oxford: Oxford University Press.

Friston, K. J., Worsley, K. J., Frackowiak, R. S. J., Mazziotta, J. C., and Evans, A. C. (1994). Assessing the significance of focal activations using their spatial extent. Hum. Brain Mapp. 1, 210-220. doi: 10.1002/hbm.460010306

Geselowitz, D. B. (1998). The zero of potential. IEEE Eng. Med. Biol. Mag. 17, 128-136. doi: 10.1109/51.646230

Gloor, P. (1985). Neuronal generators and the problem of localization in electroencephalography: application of volume conductor theory to electroencephalography. J. Clin. Psychol. 2, 327-354. doi: 10.1097/00004691-198510000-00002

Gonzalez, A., Hutchinson, J. B., Uncapher, M. R., Chen, J., Larocque, K. F., and Foster, B. L. (2015). Electrocorticography reveals the temporal dynamics of posterior parietal cortical activity during recognition memory decisions. Proc. Natl. Acad. Sci. U.S.A. 112, 11066-11071. doi: 10.1073/pnas.15107 49112

Grainger, J., and Holcomb, P. J. (2009). Watching the word go by: on the time-course of component processes in visual word recognition. Lang. Linguist. Compass 3, 128-156. doi: 10.1111/j.1749-818X.2008. 00121.x

Gramfort, A., Kowalski, M., and Matti, H. (2012). Mixed-norm estimates for the M/EEG inverse problem using accelerated gradient methods. Phys. Med. Biol. 57, 1937-1961. doi: 10.1088/0031-9155/57/7/1937

Gramfort, A., Papadopoulo, T., Olivi, E., and Clerc, M. (2010). OpenMEEG : opensource software for quasistatic bioelectromagnetics. Biomed. Eng. Online 9:45. doi: 10.1186/1475-925X-9-45
Grech, R., Cassar, T., Muscat, J., Camilleri, K. P., Fabri, S., Zervakis, M., et al. (2008). Review on solving the inverse problem in EEG source analysis. J. Neuroeng. Rehabil. 5:25. doi: 10.1186/1743-0003-5-25

Hauk, O., Davis, M. H., Ford, M., Pulvermüller, F., and Marslen-Wilson, W. D. (2006). The time course of visual word recognition as revealed by linear regression analysis of ERP data. Neuroimage 30, 1383-1400. doi: 10.1016/j.neuroimage.2005.11.048

Helenius, P., Salmelin, R., Service, E., and Connolly, J. F. (1998). Distinct time courses of word and context comprehension in the left temporal cortex Pa. Brain 121, 1133-1142. doi: 10.1093/brain/121.6.1133

Hoffman, P., and Ralph, M. A. L. (2018). Reverse concreteness effects are not a typical feature of semantic dementia : evidence for the hub-andspoke model of conceptual representation. Cereb Cortex 21, 2103-2112. doi: 10.1093/cercor/bhq288

Hofmann, M. J., Kuchinke, L., Tamm, S., Võ, M. L., and Jacobs, A. M. (2009). Affective processing within 1/10th of a second : high arousal is necessary for early facilitative. Cogn. Affect. Behav. Neurosci. 9, 389-397. doi: 10.3758/9.4.389

Huth, A. G., De Heer, W. A., Griffiths, T. L., Theunissen, F. E., and Gallant, J. L. (2016). Natural speech reveals the semantic maps that tile human cerebral cortex. Nature 532, 453-458. doi: 10.1038/nature17637

Hwang, K., Palmer, E. D., Basho, S., Zadra, J. R., and Müller, R. A. (2009). Categoryspecific activations during word generation reflect experiential sensorimotor modalities. Neuroimage 48, 717-725. doi: 10.1016/j.neuroimage.2009.06.042

Imbir, K. K., Spustek, T., and Zygierewicz, J. (2016). Effects of valence and origin of emotions in word processing evidenced by event related potential correlates in a lexical decision task. Front. Psychol. 7:217. doi: 10.3389/fpsyg.2016.00271

Kanske, P., and Kotz, S. A. (2007). Concreteness in emotional words : ERP evidence from a hemifield study. Brain Res. 1148, 138-148. doi: 10.1016/j.brainres.2007.02.044

Kawachi, Y., Kawabata, H., Kitamura, M. S., Shibata, M., Imaizumi, O., and Gyoba, J. (2011). Topographic distribution of brain activities corresponding to psychological structures underlying affective meanings : an fMRI study. Jpn. Psychol. Res. 53, 361-371. doi: 10.1111/j.1468-5884.2011.00485.x

Kemmerer, D. (2014). Cognitive Neuroscience of Language. New York, NY; London: Psychology Press.

Kiefer, M. (2001). Perceptual and semantic sources of category-specific effects : event-related potentials during picture and word categorization. Mem. Cogn. 29, 100-116. doi: 10.3758/BF03195745

Kiehl, K. A., Liddle, P. F., Smith, A. M., Mendrek, A., Forster, B. B., and Hare, R. D. (1999). Neural pathways involved in the processing of concrete and abstract words. Hum. Brain Mapp. 7, 225-233. doi: 10.1002/(SICI)10970193(1999)7:4<225::AID-HBM1>3.0.CO;2-P

Kissler, J., Assadollahi, R., and Herbert, C. (2006). Emotional and semantic networks in visual word processing: insights from ERP studies. Prog. Brain Res. 156, 147-183. doi: 10.1016/S0079-6123(06)56008-X

Koenig, T., Prichep, L., Lehmann, D., Sosa, P. V., Braeker, E., Kleinlogel, H., et al. (2002). Millisecond by millisecond, year by year: normative EEG microstates and developmental stages. Neuroimage 16, 41-48. doi: 10.1006/nimg.2002.1070

Kounios, J., and Holcomb, P. J. (1994). Concreteness effects in semantic processing: ERP evidence Supporting dual-coding theory. J. Exp. Psychol. Learn. Mem. Cogn. 20, 804-823. doi: 10.1037/0278-7393.20.4.804

Kousta, S., Vigliocco, G., Vinson, D. P., Andrews, M., and Del Campo, E. (2011). The representation of abstract words : why emotion matters. J. Exp. Psychol. Gen. 140, 14-34. doi: 10.1037/a0021446

Kuchinke, L., Jacobs, A. M., Grubich, C., Võ, M. L., Conrad, M., and Herrmann, M. (2005). Incidental effects of emotional valence in single word processing : an fMRI study. Neuroimage 28, 1022-1032. doi: 10.1016/j.neuroimage.2005.06.050

Kutas, M. (1993). In the company of other words: electrophysiological evidence for single-word and sentence context effects. Lang. Cogn. Process. 8, 533-572. doi: 10.1080/01690969308407587

Kutas, M., and Federmeier, K. D. (2011). Thirty years and counting : finding meaning in the N400 component of the event-related brain potential (ERP). Annu. Rev. Psychol. 62, 621-647. doi: 10.1146/annurev.psych.093008.131123

Lambon Ralph, M. A. (2013). Neurocognitive insights on conceptual knowledge and its breakdown. Philos. Trans. R. Soc. Lond. B Biol. Sci. 369, 20120392. doi: 10.1098/rstb.2012.0392

Laszlo, S., and Federmeier, K. D. (2014). Never seem to find the time: evaluating the physiological time course of visual word recognition with 
regression analysis of single item ERPs. Lang. Cogn. Process 29, 642-661. doi: 10.1080/01690965.2013.866259

Lau, E. F., Phillips, C., and Poeppel, D. (2008). A cortical network for semantics: (de)constructing the N400. Nat. Rev. Neurosci. 9, 920-933. doi: $10.1038 / \mathrm{nrn} 2532$

Lewis, P. A., Critchley, H. D., Rotshtein, P., and Dolan, R. J. (2007). Neural correlates of processing valence and arousal in affective words. Cereb Cortex. 17, 742-748. doi: 10.1093/cercor/bhk024

Luck, S. J., and Gaspelin, N. (2017). How to get statistically significant effects in any ERP experiment ( and why you shouldn 't ). Psychophysiology 54, 146-157. doi: $10.1111 /$ psyp.12639

Macdonald, A. W., Cohen, J. D., Stenger, A. V., and Carter, C. S. (2000). Dissociating the role of the dorsolateral prefrontal and anterior cingulate cortex in cognitive control. Science 288, 1835-1838. doi: $10.1126 /$ science.288.5472.1835

Mahjoory, K., Nikulin, V. V., Botrel, L., Linkenkaer-hansen, K., Fato, M. M., and Haufe, S. (2017). Consistency of EEG source localization and connectivity estimates. Neuroimage 152, 590-601. doi: 10.1016/j.neuroimage.2017.02.076

Mahon, B. Z., and Caramazza, A. (2008). A critical look at the embodied cognition hypothesis and a new proposal for grounding conceptual content. J. Physiol. 102, 59-70. doi: 10.1016/j.jphysparis.2008.03.004

Marian, V., Bartolotti, J., Chabal, S., and Shook, A. (2012). Clearpond: cross-linguistic easy-access resource for phonological and orthographic neighborhood densities. PLoS ONE 7:e43230. doi: 10.1371/journal.pone.0043230

Marí-beffa, P., Catena, A., Valdés, B., Cullen, D., and Houghton, G. (2007). $\mathrm{N} 400$, the reference electrode, and the semantic activation in prime-task experiments: a reply to Dombrowski and Heil (2006). Brain Res. 1147, 209-212. doi: 10.1016/j.brainres.2007.01.124

Martin, A., and Chao, L. L. (2015). Semantic memory and the brain : Structure and processes Semantic memory and the brain : structure and processes. Curr. Opin. Neurobiol. 11, 194-201. doi: 10.1016/S0959-4388(00)00196-3

Michel, C. M., Murray, M. M., Lantz, G., Gonzalez, S., Spinelli, L., and Grave De Peralta, R. (2004). EEG source imaging. Clin. Neurophysiol. 115, 2195-2222. doi: 10.1016/j.clinph.2004.06.001

Moors, A., De Houwer, J., Hermans, D., Winne, J., and De, Brysbaert, M. (2013). Norms of valence, arousal, dominance, and age of acquisition for 4, 300 Dutch words. Behav. Res. Methods 45, 169-177. doi: 10.3758/s13428-0120243-8

Nobre, a. C., and McCarthy, G. (1995). Language-related field potentials in the anterior-medial temporal lobe: II. Effects of word type and semantic priming. J. Neurosci. 15, 1090-1098. doi: 10.1523/JNEUROSCI.15-02-01090. 1995

Nunez, P. L., and Srinivasan, R. (2006). Electric Fields of the Brain: The Neurophysics Of EEG. New York, NY: Oxford University Press.

Osgood, C. E., May, W. H., and Miron, M. S. (1975). Cross-Cultural Universals of Affective Meaning. Vol. 1. Urbana, IL; Chicago, IL; London: University of Illinois Press.

Palazova, M., Sommer, W., and Schacht, A. (2013). Brain \& Language Interplay of emotional valence and concreteness in word processing : an event-related potential study with verbs. Brain Lang. 125, 264-271. doi: 10.1016/j.bandl.2013.02.008

Pascual-Marqui, R. D. (2002). Standardized low-resolution brain electromagnetic tomography (sLORETA): technical details. Methods Find. Exp. Clin. Pharmacol. 24, 5-12.

Pascual-marqui, R. D. (2007). Discrete, 3D distributed linear imaging methods of electric neuronal activity. Part 1: exact, zero error localization. arXiv:0710.3341.
Perani, D., Cappa, S. F., Schnur, T., Tettamanti, M., Collina, S., Rosa, M., et al. (1999). The neural correlates of verb and noun processing A PET study. Brain 122, 2337-2344. doi: 10.1093/brain/122.12.2337

Price, C. J. (2015). The anatomy of language: contributions from functional neuroimaging. J. Anat. 44, 335-359. doi: 10.1046/j.1469-7580.2000.19730335.x

Ptak, R. (2012). The frontoparietal attention network of the human brain: action, saliency, and a priority map of the environment. Neuroscience 18, 502-515. doi: $10.1177 / 1073858411409051$

Schaefer, M., and Rotte, M. (2010). Combining a semantic differential with fMRI to investigate brands as cultural symbols. Soc. Cogn. Affect. Neurosci. 5, 274-281. doi: $10.1093 /$ scan/nsp055

Silva-pereyra, J., Rivera-Gaxiola, M., Aubert, E., Bosch, J., Galan, L., and Salazar, A. (2003). N400 during lexical decision tasks : a current source localization study. Clin. Neurophysiol. 114, 2469-2486. doi: 10.1016/S1388-2457(03)00248-7

Simanova, I., Gerven, M., Van Oostenveld, R., and Hagoort, P. (2010). Identifying object categories from event-related EEG: toward decoding of conceptual representations. PLoS ONE 5:e14465. doi: 10.1371/journal.pone.0014465

Skrandies, W. (1998). Evoked potential correlates of semantic meaning-A brain mapping study. Cogn. Brain Res. 6, 173-183. doi: 10.1016/S0926-6410(97)00033-5

Song, J., Davey, C., Poulsen, C., Luu, P., Turovets, S., Anderson, E., et al. (2015). EEG source localization : sensor density and head surface coverage. J. Neurosci. Methods 256, 9-21. doi: 10.1016/j.jneumeth.2015.08.015

Tadel, F., Baillet, S., Mosher, J. C., Pantazis, D., and Leahy, R. M. (2011). Brainstorm : a user-friendly application for MEG/EEG analysis. Comput. Intell. Neurosci. 13: 879716. doi: 10.1155/2011/879716

Tsao, D. Y., Moeller, S., and Freiwald, W. A. (2008). Comparing face patch systems in macaques and humans. Proc. Natl. Acad. Sci. U.S.A. 105, 19514-19519. doi: 10.1073/pnas.0809662105

Verbeke, G., and Molenberghs, G. (2000). Linear Mixed Models for Longitudinal Data. Springer: Springer, 19-26.

Vigliocco, G., Kousta, S., Anthony, P., Rosa, D., Vinson, D. P., Tettamanti, M., et al. (2014). The neural representation of abstract words : the role of emotion. Cereb Cortex. 24, 1767-1777. doi: 10.1093/cercor/bht025

Wang, J., Baucom, L. B., and Shinkareva, S. V. (2013). Decoding abstract and concrete concept representations based on single-trial fMRI data. Hum. Brain Mapp. 34, 1133-1147. doi: 10.1002/hbm.21498

Wang, J., Conder, J. A., Blitzer, D. N., and Shinkareva, S. V. (2010). Neural representation of abstract and concrete concepts : a metaanalysis of neuroimaging studies. Hum. Brain Mapp. 1468, 1459-1468. doi: 10.1002/hbm.20950

West, W. C., and Holcomb, P. J. (2000). Imaginal, semantic, and surfacelevel processing of concrete and abstract words : an electrophysiological investigation. J. Cogn. Neurosci. 12, 1024-1037. doi: 10.1162/0898 9290051137558

Conflict of Interest Statement: The authors declare that the research was conducted in the absence of any commercial or financial relationships that could be construed as a potential conflict of interest.

Copyright (C) 2018 Fahimi Hnazaee, Khachatryan and Van Hulle. This is an openaccess article distributed under the terms of the Creative Commons Attribution License (CC BY). The use, distribution or reproduction in other forums is permitted, provided the original author(s) and the copyright owner(s) are credited and that the original publication in this journal is cited, in accordance with accepted academic practice. No use, distribution or reproduction is permitted which does not comply with these terms. 